\title{
Evidence for terahertz acoustic phonon parametric oscillator based on acousto-optic degenerate four-wave mixing in a silicon doping superlattice
}

\author{
Thomas E. Wilson* \\ Department of Physics, Marshall University, Huntington, West Virginia 25755, USA
}

(Received 12 August 2018; revised manuscript received 21 November 2018; published 28 December 2018)

\begin{abstract}
We report evidence for a 1.0-THz self-starting mirrorless acoustic phonon parametric oscillator (MAPPO) produced from acousto-optic phase-conjugate degenerate four-wave (D4WM) mixing in a THz laser-pumped silicon doping superlattice (DSL). The DSL was grown by molecular beam epitaxy on a (100) boron-doped silicon substrate. A superconducting NbTiN subwavelength grating was used to couple the $\mathrm{THz}$ laser radiation into the DSL. Superconducting granular aluminum bolometric detection, coupled with Si:B piezophonon spectroscopy, revealed excitation of $\mathrm{THz}$ coherent compressional and shear waves, along the $\langle 111\rangle$ direction only. The Bragg scattering condition for distributed feedback, and the energy conservation requirement for the D4WM process, were both verified. A THz MAPPO could provide a test bed for studies of nonclassical acoustic phonon fields.
\end{abstract}

DOI: 10.1103/PhysRevB.98.220304

Phonons, the quanta associated with the vibrational waves of a crystalline lattice, are among the most important excitations in condensed matter. Major advances in our understanding of the physics of phonons during the past several decades have included first-principles calculations of the phonon lifetimes in simple crystals, their interactions with other phonons, defects, boundaries, and impurities, and in controlling thermal transport at the nanoscale [1]. A coherent source of acoustic phonons could find widespread applications in science and technology. The stimulated emission of transverse acoustic (TA) phonon avalanches at $50.4 \mathrm{GHz}$ has been observed in dilute centers in ruby [2]. Current phonon studies use visiblelight pulse (VLP) transducer techniques [3] to produce and detect coherent near-THz acoustic phonons. In most VLPbased experiments, the generation of coherent phonons was limited to longitudinal acoustic (LA) phonons because of the lateral symmetry of the excited crystal, although TA phonons at $0.4 \mathrm{THz}$ have also been produced by piezoelectric coupling in GaN multiple quantum wells [4]. A (femto- or picosecond) VLP produces, by electronic excitation and relaxation, a stress as a source of near-THz coherent acoustic phonons. Stress joined with an LA or TA wave can be monitored by a pumpprobe technique measuring changes in reflectivity. A VLP transducer can consist of a metal film, a surface region of a crystal, or a superlattice (SL). More recently, the sound amplification by stimulated emission of radiation (saser) in semiconductor superlattice structures has been described in connection with the VLP transducer technique. One type of saser uses an active medium, a population inversion in a voltage-biased SL containing free electrons [5,6]. Gain is due to phonon stimulated electronic transitions between next-nearest energy levels of a Wannier-Stark ladder; the gain coefficient for LA waves at $650 \mathrm{GHz}$ was $3 \times 10^{3} \mathrm{~cm}^{-1}$. Another type [7] utilizes a miniband current in a SL (at

*Corresponding author: wilsont@marshall.edu room temperature) showing gain due to the acoustoelectric effect; its gain coefficient for LA waves near $400 \mathrm{GHz}$ was $8 \times 10^{3} \mathrm{~cm}^{-1}$.

In this Rapid Communication, we report experimental evidence for a mirrorless acoustic phonon parametric oscillator (MAPPO) in a doping superlattice structure operating at $1 \mathrm{THz}$. It is based upon acousto-optic (AO) phase-conjugate degenerate four-wave mixing (4WM) [8] of counterpropagating fields. The MAPPO is optically pumped by nanosecond (ns)-pulsed 1.04-THz plane-polarized laser radiation. Coherent light sources based upon 4WM in mirrorless laser oscillators, in both hot and cold atoms [9], are known to have some unique properties such as cavity-free alignment and large tunability. We have designed the SL period to provide spatially distributed feedback. Distributed feedback occurs when the Bragg scattering condition is satisfied for the signal and phase-conjugate acoustic waves propagating normal to a gain grating [10] associated with the 4WM process. We have not, however, made any first-principles calculations, and more data are necessary to further validate, and more fully understand, the phenomenon.

A " $n-i-p-i$ " DSL [11] is formed by a periodic $n$ - and $p$ doping structure in an otherwise homogeneous semiconductor crystal with undoped $(i)$ regions in between. In its ground state the sample thus consists of a single crystal (in contrast to compositional SLs) with alternately positively and negatively charged sheets of ions. Given the backfolded acoustic phonons (see Fig. 1 of Ruden and Dohler [12]) under the superlattice aspect are optical modes, doping SLs could be expected to be excitable by far-infrared (FIR) electromagnetic fields. Our delta-doped doping SLs were grown by molecular beam epitaxy (MBE) on (100) float-zone 150-mm-diam silicon wafers and consisted of 30 sequential $n-i-p-i$ 's with period $d=8.1 \mathrm{~nm}$ and dopant (B and $\mathrm{Sb}$ ) concentrations: $N_{A}^{2 \mathrm{D}}=$ $N_{D}^{2 \mathrm{D}}=3.3 \times 10^{13} \mathrm{~cm}^{-2}$. The smallest subband energy level spacing in the $\mathrm{V}$-shaped potential wells for both electrons and holes is of order $50 \mathrm{meV}$ in our doping SL [13], much 


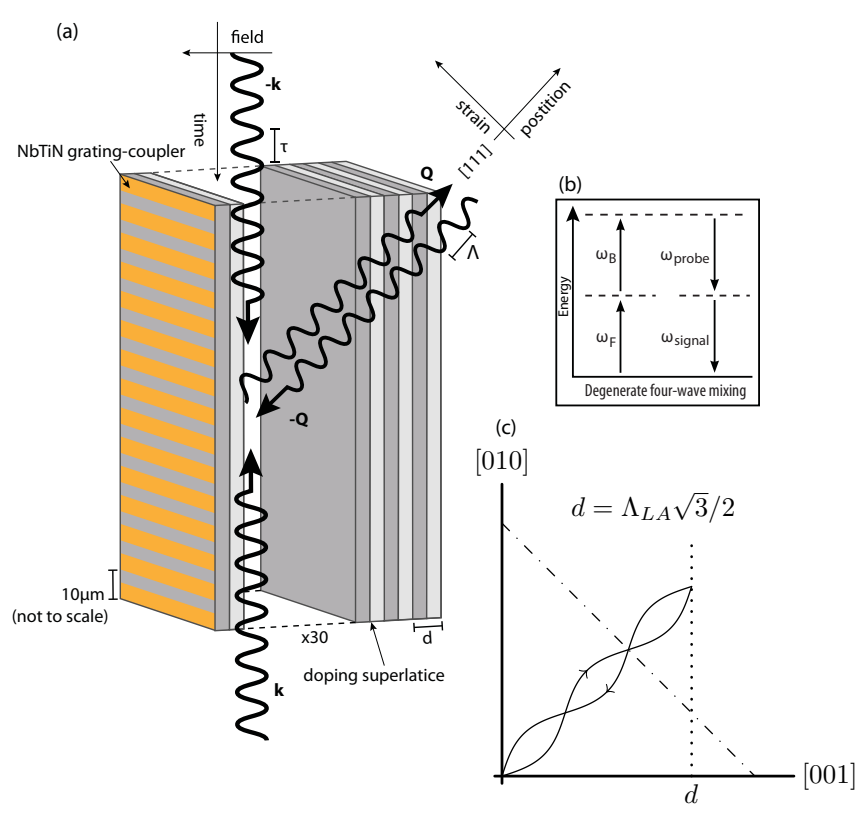

FIG. 1. (a) Schematic diagram of MAPPO showing $k /-k$ evanescent electromagnetic fields, and $\langle 111\rangle$-directed $Q /-Q$ (PC) acoustic fields. (b) Energy conservation diagram for D4WM. (c) Distributedfeedback phase matching, projected onto (100) plane, results in a standing LA wave (solid lines) for $Q /-Q$. DSL (dotted line) of period $d$. Gain grating (dashed-dotted line) shown. LA wavelength: $\Lambda_{\mathrm{LA}}$.

larger than the 4.3-meV pump radiation. Intense $\mathrm{THz}$ fields on the order of $\mathrm{MV} / \mathrm{cm}$ have been reported to induce carrier generation by impact ionizations associated with impurity states in undoped multiple quantum wells [14], however, in our experiments we use quite modest $\mathrm{THz}$ fields on the order $10 \mathrm{~V} / \mathrm{cm}$. Accordingly, our AO 4WM process should lie in the reactive regime [15] where the FIR light frequency is below the onset of absorption by electronic transitions.

Acoustic phase conjugation has been observed in piezoelectric semiconductors at microwave frequencies [16] resulting from a nonlinear piezoelectric coupling between photons and phonons (or electric field $E$ and elastic strain $S$ ) of the form $\gamma E^{2} S^{2}$, with $\gamma$ the coupling constant. This fourwave coupling is of the same form as that found in optical 4WM giving rise to optical phase conjugation. We postulate that electrostriction coupling of this form is present in our experiments due to the direct action of the electric field acting upon the ion sheets of the DSL. Recently, electrostriction has also been reported to have produced an extraordinarily strong D4WM response yielding $\mathrm{THz}$ acoustic breathing modes in gold nanoparticles [17]. Figure 1 illustrates schematically our posited 1.04-THz MAPPO process (energy conservation and distributed feedback have been verified as described below). In AO D4WM one of the optical pump waves and the acoustic probe wave couple to produce an index grating [18]. This grating coherently scatters the counterpropagating optical pump wave to produce a phase-conjugate acoustic wave. Distributed feedback coupled with parametric gain can then resonantly generate a standing-wave pattern along $\langle 111\rangle$. The index gain-grating planes are normal to the acoustic wave

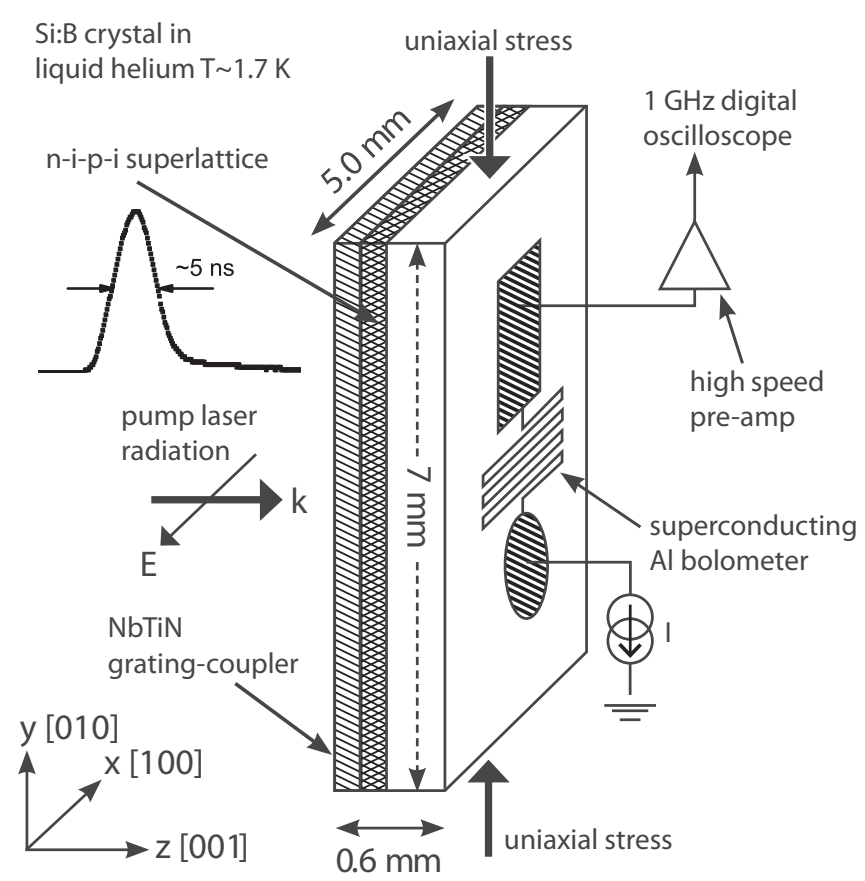

FIG. 2. Schematic diagram of experimental arrangement. Thicknesses of grating coupler and DSL (cross-hatched) not to scale. THz pump laser radiation incident from left with wave vector $k, E$ field, and typical digitized pulse envelope shown.

vector. In contrast to optical parametric oscillators (OPOs), for MAPPOs the difficulties of phase matching due to dispersion are largely circumvented [15].

Figure 2 illustrates our experimental arrangement. A silicon crystal (size $7 \mathrm{~mm} \times 5 \mathrm{~mm} \times 0.60 \mathrm{~mm}$ ) is immersed in vapor-pumped liquid helium $(1.66 \mathrm{~K})$ and carries on one surface a superconducting NbTiN grating coupler patterned on top of the doping SL, and on the opposite crystal surface a superconducting $\mathrm{Al}$ bolometer. The grating coupler is illuminated by nanosecond-pulsed polarized $1.04-\mathrm{THz}$ laser radiation. We chose to use NbTiN for the grating coupler (500 $\mathrm{nm}$ thick, $10 \mu \mathrm{m}$ period, $6 \mu \mathrm{m}$ line, $4 \mu \mathrm{m}$ space) due to its high-frequency gap $1.2 \mathrm{THz}$ [19] and low absorptivity, to preclude the generation of broadband incoherent acoustic phonons. The subwavelength grating coupler converts the incident FIR electromagnetic wave into counterpropagating evanescent surface waves within the DSL as shown in Fig. 1(a). Grating-coupler theory [20] shows that $85 \%$ of the incident FIR laser intensity is converted into the surface waves with $15 \%$ remaining in the propagating field. The evanescent field, with a $1.7-\mu \mathrm{m}$ decay length, can be assumed to be uniform over the $0.24-\mu \mathrm{m}$-thick DSL, and does not extend appreciably into the $0.60-\mathrm{mm}$-thick Si:B substrate. The 100$\mathrm{nm}$-thick serpentine bilayer granular aluminum/palladium bolometer [21], with an active area $A=0.53 \mathrm{~mm}^{2}$, was used for the detection of both photons and phonons. The phaseconjugate acoustic wave would not be detected. The bolometer's responsivity $\mathcal{R}=900 \mathrm{~V} / \mathrm{W}$ was obtained through a combined dc and ns-pulsed characterization study [22]. The bolometer was biased at a constant current of $40 \mu \mathrm{A}$, and the voltage signal was amplified $40 \times$ using $1-\mathrm{GHz}$ bandwidth 


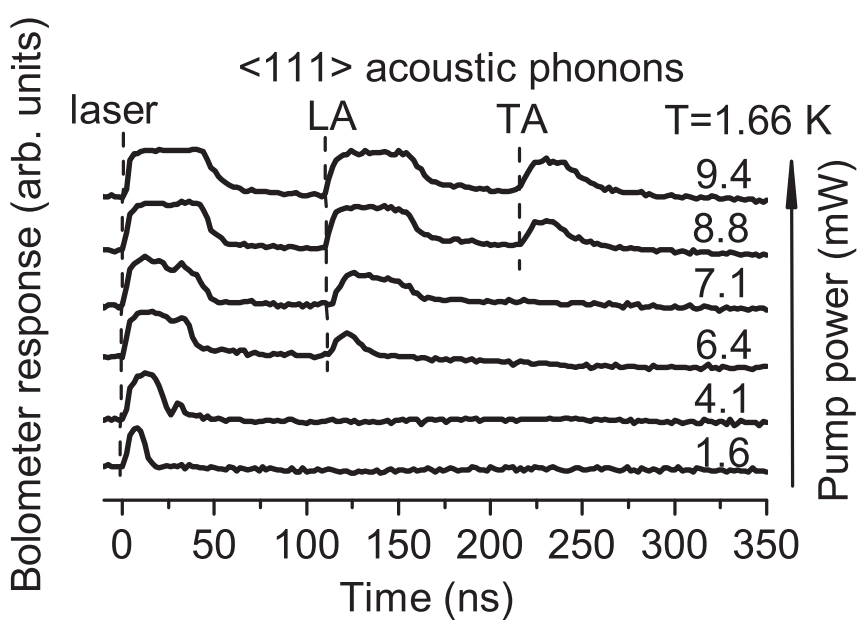

FIG. 3. Stacked sequence of digitized bolometer signals (arb. units) for increasing (estimated) pump power ( $\mathrm{mW}$ ) listed above traces.

electronics, and recorded using a fast real-time digital sampling oscilloscope.

The $\mathrm{Si}$ substrate was boron doped at a density of $N_{\mathrm{B}}=$ $10^{15} \mathrm{~cm}^{-3}$ in order to use Si:B piezophonon spectroscopy [23,24]. The resonant phonon scattering by the stress-split acceptor ground state can be used for phonon spectroscopy. We chose the boron doping density $N_{\mathrm{B}}$ appropriate for our sample thickness, according to a scaling law found in the Appendix of Schwarte and Berberich [25], $N_{\mathrm{B}} \sim N_{\mathrm{SB}} \frac{4 \mathrm{~mm}}{0.6 \mathrm{~mm}}$, where $N_{\mathrm{SB}}=4.8 \times 10^{13} \mathrm{~cm}^{-3}$ and $4 \mathrm{~mm}$ were their doping level and sample thickness, respectively. The scattering rate for TA phonons was shown [25] to be larger than for LA phonons, although the respective scattering rates approach each other near $5 \mathrm{meV}$. We used a uniaxial stress apparatus designed after Fröhlich and Nieswand [26], and calibrated up to $3 \mathrm{kbar}$ to apply the stress via a spring balance mounted above the cryostat.

We used a previously described [27] custom cavitydumped, optically pumped, FIR molecular gas laser to pump the DSL. The FIR laser resonator, filled with methyl fluoride at a pressure of 6 Torr and pumped by the 9R20 line of a multimode transversely excited atmospheric $\mathrm{CO}_{2}$ laser, yielded smooth cavity-dumped pulse envelopes [ $\Delta t \simeq 5 \mathrm{~ns}$ (full width at half maximum, FWHM)] (see Fig. 2), as measured by a $1-\mathrm{GHz}$ bandwidth pyroelectric detector, containing the linearly polarized $1.04-\mathrm{THz}$ radiation. Peak powers of order $10 \mathrm{~kW}$ were obtained at $10-\mathrm{Hz}$ pulse repetition rates. The measured wavelength, $\lambda=288.2 \pm 0.05 \mu \mathrm{m}$, obtained with a scanning metal-mesh (1000-lpi) Fabry-Pérot interferometer [28], agreed with the published wavelength [29] for this FIR line. The energy contained in a FIR pulse fluctuated by $\approx 50 \%$ from pulse to pulse, with frequent larger excursions. A waveguide [30], used for FIR laser beam transport to the entrance window of the liquid-helium cryostat, was interrupted to insert a pair of crossed wire-grid polarizers to reduce the pump power to $\sim 10 \mathrm{~mW}$.

Figure 3 shows a stacked sequence of digitized bolometer traces (signal versus time) in response to single laser pulses of progressively increasing power. Due to the nonlinearity of the bolometer response (described below), however, the listed powers are suspect. The first pulse observed (time $0 \mathrm{ns)}$ in all traces is the bolometer response to the arrival of undiffracted grating-coupled FIR laser radiation. As the laser radiation pump power reaches a threshold power of $6.4 \mathrm{~mW}$, ballistic acoustic phonons appear at a delay time of $110 \pm 2 \mathrm{~ns}$. As the incident FIR power increases further, the acoustic signal at the 110-ns delay grows progressively stronger, and at an 8.8-mW pump power, a second phonon signal appears at $210 \pm 2$ ns. The distinct onsets of the phonon signals with pump power may be misleading, however, due to the nonlinearity of the bolometer response. For the determination of group velocities of waves consistent with observed signals, we use the equations of motion (Christoffel's equations [31]) for sound waves in silicon (mass density $\rho=2.332 \mathrm{~g} / \mathrm{cm}^{3}$, elastic constants $c_{11}=167.8 \mathrm{GPa}, c_{12}=65.2 \mathrm{GPa}, c_{44}=$ $80.01 \mathrm{GPa}$ [ [32]. Among the surveyed modes for $\langle 100\rangle,\langle 110\rangle$, $\langle 111\rangle,\langle 012\rangle$, and $\langle 122\rangle$ directions, we find agreement with the measured transit times across the 0.60-mm-thick (100) Si:B substrate only for the $\langle 111\rangle$ LA and TA modes. The group (and phase) velocities for the $\langle 111\rangle \mathrm{LA}$ and TA modes are $v_{\mathrm{LA}}=$ $9.400 \mathrm{~km} / \mathrm{s}$ and $v_{\mathrm{TA}}=5.110 \mathrm{~km} / \mathrm{s}$, respectively. The computed transit times for the $\langle 111\rangle$ LA and TA modes are 110 and $203 \mathrm{~ns}$, in good agreement with the experimental values, 110 and $210 \mathrm{~ns}$, respectively (Fig. 3). The slight discrepancy with the TA transit time may be due to a change in the elastic constants due to the $\mathrm{Si}: \mathrm{B}$ substrate doping. Identical arrival times, however, would be expected for phonons produced along any of the four equivalent $\langle 111\rangle$ forward directions. The Bragg scattering condition for distributed feedback [see Fig. 1(c)] is given by

$$
2 Q \cos (\theta)=N G
$$

with $\theta$ the angle between the phonon wave vector $\vec{Q}$ and the DSL reciprocal lattice vector $\vec{G}=\frac{2 \pi}{d}[001]$, and with $N$ integer. For the observed $\langle 111\rangle$ LA and TA modes at $1.04 \mathrm{THz}$ (frequency verified below), with associated wavelengths $\Lambda_{\mathrm{LA}}=9.35 \mathrm{~nm}$ and $\Lambda_{\mathrm{TA}}=4.91 \mathrm{~nm}$, respectively, we indeed find near integer values $N$ for both LA and TA modes, i.e., 1.00 and 1.90 , respectively. Thus, the experimental results are in excellent agreement with the distributed feedback requirement. A higher threshold observed for the TA mode could be expected since the Bragg scattering condition is not quite as well satisfied in this case, i.e., 1.90 instead of 2.00 . Interestingly, a [001] LA mode at $1.04 \mathrm{THz}$ would also satisfy the Bragg condition with $N=1.00$, but this mode is not observed.

As shown in Fig. 3, as the incident FIR estimated power increases, the registered bolometer signals broaden in time and the amplitudes saturate. Thus, the bolometer unfortunately was operated in a nonlinear regime in these experiments. To continue with an analysis, we will assume that the integrated area of a given pulse is proportional to the absorbed energy. We accordingly assume that the absorbed signal power is proportional to its integrated pulse area, relative to that of the integrated weakest laser pulse response (lowest trace in Fig. 3). Estimated absorbed powers were then obtained by multiplying the ratio of areas by the peak voltage of the weak laser pulse, and dividing by the bolometer responsivity $\mathcal{R}$ 


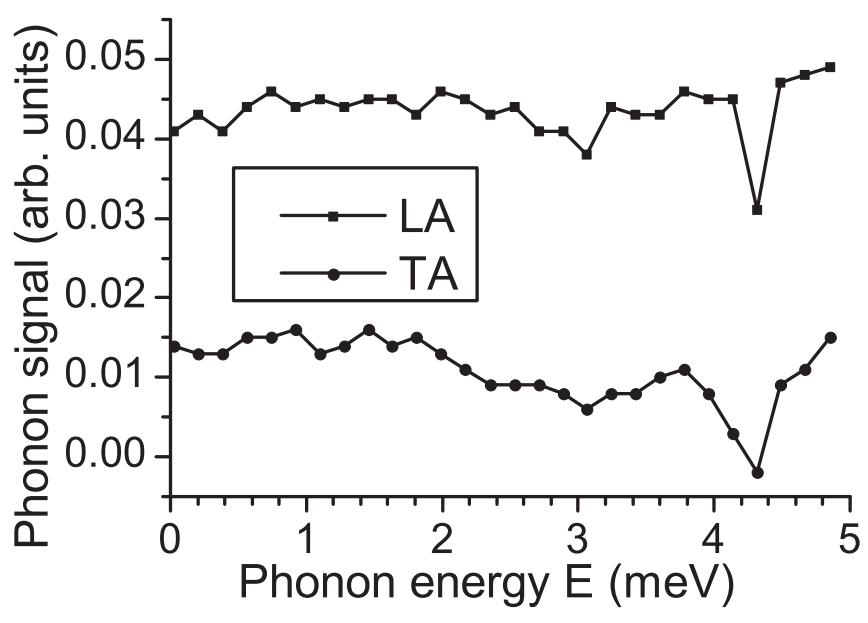

FIG. 4. LA (top) and TA (bottom) phonon signals (points with lines to guide the eye) vs applied [100] uniaxial stress, or phonon energy $\left(2.73 \times 10^{-3} \mathrm{meV} /\right.$ bar $) .0 .18-\mathrm{meV}$ step size.

and the postdetection electronics amplification. The estimated incident pump powers are obtained (numbers shown above traces to the right in Fig. 3) from the absorbed FIR pump powers by dividing by 0.15 (that fraction of the zero-order propagating light reaching the bolometer) and by 0.016 , the FIR absorbtance of the bolometer. The latter was calculated using the theory of electromagnetic wave propagation in stratified dielectric and metallic media [33], using the lowtemperature FIR index of refraction of silicon [34], 3.318, and the resistivity, $235 \mu \Omega \mathrm{cm}$, of the biased bolometer. Estimates of the acoustic pulse power within the doping DSL result from dividing the bolometer's absorbed acoustic power by 0.30 , the estimated acoustic wave absorptance of the bolometer. The latter is consistent with dielectric crystal/metal interface studies [35].

Figure 4 displays separately the LA and TA phonon spectra as obtained by $\mathrm{Si}$ :B piezophonon spectroscopy. The averaged $\left(10^{3}\right.$ single shots) peak of a bolometer response to a phonon pulse, for a time gate of width $\Delta t$ positioned at the arrival time for the mode in question, is plotted versus the Si:B resonant scattering energy $E$. Here, the peak value of each bolometer phonon signal, prior to averaging, was divided by the peak value of a separately detected portion of the FIR pump radiation that reflected from a wire-mesh beam splitter and focused onto a pyroelectric detector. As a result, the averaged bolometer responses shown are in arbitrary units, and the corresponding pump power was not estimated from the bolometer response in this case. The phonon energies for both LA and TA signals (the drop in the signals) are identified as $4.3 \mathrm{meV}$, corresponding to $1.04 \mathrm{THz}$, in excellent agreement with the presumed AO D4WM process. On average, the TA signal is weaker $\left(\times \frac{1}{4}\right)$. It is well known from phonon focusing studies in silicon [36] using incoherent sources that TA phonon signals are stronger than LA signals along $\langle 111\rangle$. Since our data shown in Figs. 3 and 4 indicate the opposite, we have further evidence for coherent, and not incoherent, phonon generation.

Figure 5 shows a plot of the estimated LA power versus the 1.04- THz estimated pump power. Here, we have divided the

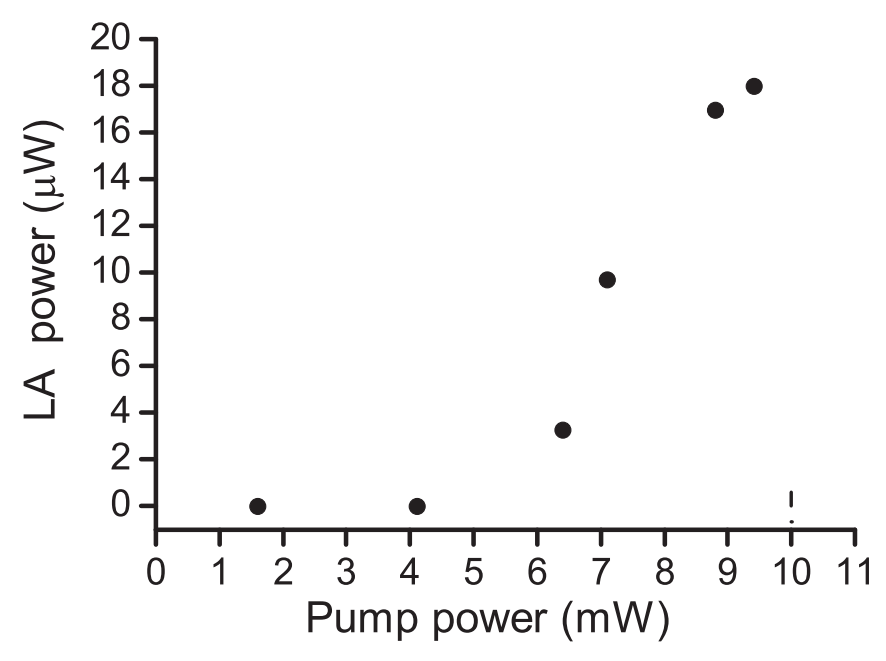

FIG. 5. Estimated LA power vs the estimated pump power of the FIR laser radiation.

full incident LA power by 4 to account for power assumed to emanate from four $\langle 111\rangle$ equivalent directions. For the pump power $P \sim 9.4 \mathrm{~mW}$ and estimated $\langle 111\rangle$ LA power $\sim 18 \mu \mathrm{W}$, we find a conversion efficiency, $\eta \sim 2 \times 10^{-3}$. The propagating acoustic pulse contains $9 \times 10^{-14} \mathrm{~J}$ in a volume $V=v_{\mathrm{LA}} \Delta t A / \sqrt{3}=1.4 \times 10^{-11} \mathrm{~cm}^{3}$. The estimated phonon occupation number in this volume is $n=1.4 \times 10^{8}$. The zero-point amplitude [37] is given by $\zeta_{\mathrm{zp}}=\sqrt{\hbar \rho V^{*} \omega} \sim$ $3.2 \times 10^{-18} \mathrm{~m}$, where we choose for the initial coherence volume $V^{*} \sim 1.4 \times 10^{-15} \mathrm{~m}^{3}$, a cylinder formed by the circular projected bolometer area $A / \sqrt{3}$, and a coherence length $\Lambda_{\mathrm{LA}} / 2$ (due to elastic scattering at $\mathrm{B}$ and $\mathrm{Sb}$ impurities). The avalanche of stimulated LA phonons then corresponds to a classical acoustic wave of amplitude $\zeta=\zeta_{\text {zp }} \sqrt{n+1} \sim$ $3.7 \times 10^{-14} \mathrm{~m}$. This amplitude corresponds to $2.7 \times 10^{-4}$ of the distance between an impurity and the nearest $\mathrm{Si}$ atom, or strain, $S \sim 3 \times 10^{-4}$. Picosecond acoustic solitons formed in Si have reported strain levels of this order of magnitude [38]. For an active medium length of $L=30 \times d \times \sqrt{3}=$ $0.42 \mu \mathrm{m}$, and assuming a unity zero-point fluctuation, we find a large exponential gain coefficient, $g_{\mathrm{LA}}=\ln (n / 1) / L \simeq$ $4 \times 10^{5} \mathrm{~cm}^{-1}$.

In summary, we report evidence for a self-starting MAPPO operating at $1.04 \mathrm{THz}$. A MAPPO may prove useful as a source of coherent acoustic phonons with both compressional and shear waves available for probing material properties at the nanoscale. In principle, a MAPPO might also be operated in reverse [18] as an acoustic wave detector; coherent incident acoustic waves could excite coherent FIR radiation which could be coupled out using a grating or a prism. Given that stimulated optical 3WM and 4WM have been used to study quantum correlations between photon beams $[9,39,40]$, nonlinear MAPPOs could also provide a test bed for analogous studies of nonclassical acoustic phonon fields.

I wish to extend my appreciation for fruitful discussions to K. F. Renk, to E. Kasper, J. Schulze, and M. Oehme for providing the DSL samples, to A. Lichtenberger for the 
NbTiN grating fabrication, and to K. A. Korolev and S. M. Barber for technical assistance. We acknowledge funding from NASA EPSCoR Grant No. NNX11AM04A, AFOSR Grant No. FA9550-12-1-0100, West Virginia Higher Educa- tion Policy Commission Grant No. HECP.DSR.12.129, and the use and staff support of both the West Virginia University Shared Resource Facility and the Marshall University Molecular and Biological Imaging Center.
[1] D. G. Cahill, P. V. Braun, G. Chen, D. R. Clarke, S. Fan, K. E. Goodson, P. Keblinski, W. P. King, G. D. Mahan, A. Majumdar, H. J. Maris, S. R. Phillpot, E. Pop, and L. Shi, Appl. Phys. Rev. 1, 011305 (2014).

[2] L. G. Tilstra, A. F. M. Arts, and H. W. de Wijn, Phys. Rev. B 68, 144302 (2003).

[3] P. Ruello and V. E. Gusev, Ultrasonics 56, 21 (2015).

[4] C.-C. Chen, H.-M. Huang, T.-C. Lu, H.-K. Kuo, and C.-K. Sun, Appl. Phys. Lett. 100, 201905 (2012).

[5] W. Maryam, A. V. Akimov, R. P. Campion, and A. J. Kent, Nat. Commun. 4, 2184 (2013).

[6] A. J. Kent and R. Beardsley, in Length-Scale Dependent Phonon Interactions, edited by S. L. Shindé and P. Shrivastava (Springer, New York, 2014), Chap. 8.1, p. 227.

[7] K. Shinokita, K. Reimann, M. Woerner, T. Elsaesser, R. Hey, and C. Flytzanis, Phys. Rev. Lett. 116, 075504 (2016).

[8] A. Yariv and D. M. Pepper, Opt. Lett. 1, 16 (1977).

[9] Y. Mei, X. Guo, L. Zhao, and S. Du, Phys. Rev. Lett. 119, 150406 (2017).

[10] M. J. Damzen, in Phase Conjugate Laser Optics, edited by A. Brignon and J.-P. Huigard (Wiley, Hoboken, NJ, 2004), Chap. 11.8 , p. 390.

[11] G. H. Dohler, Crit. Rev. Solid State Mater. Sci. 13, 97 (1986).

[12] P. Ruden and G. H. Dohler, Solid State Commun. 45, 23 (1983).

[13] H.-J. Gossmann and E. F. Shubert, Crit. Rev. Solid State Mater. Sci. 18, 1 (1993).

[14] K. Shinokita, H. Hirori, K. Tanaka, T. Mochizuki, C. Kim, H. Akiyama, L. N. Pfeiffer, K. W. West, Phys. Rev. Lett. 111, 067401 (2013).

[15] C. Flytzanis, in Nonlinear Acoustics - Fundamentals and Applications: 18th International Symposium on Nonlinear Acoustics - ISNA 18, edited by B. Enflo, C. M. Hedberg, and L. Kari, AIP Conf. Proc. Vol. 1022 (AIP, Melville, NY, 2008), p. 471.

[16] K. Fossheim, in Nonequilibrium Phonon Dynamics, edited by W. E. Bron, NATO Advanced Studies Institute, Series B: Physics (Plenum, New York, 1984), Vol. 124, p. 277.

[17] D. Xiang, J. Wu, J. Rottler, and R. Gordon, Nano Lett. 16, 3638 (2016).

[18] D. Rogovin, Phys. Rev. A 41, 6805 (1990).

[19] B. Leone, B. D. Jackson, J. R. Gao, and T. M. Klapwijk, Appl. Phys. Lett. 76, 780 (2000).

[20] W. J. Li and B. D. McCombe, J. Appl. Phys. 71, 1038 (1992).

[21] T. E. Wilson, K. A. Korolev, and N. A. Crow, J. Micro/Nanolithogr., MEMS, MOEMS 14, 014501 (2015).
[22] B. A. Danilchenko, C. Z. Jasiukiewicz, T. Paszkiewicz, and S. Wolksi, Acta Phys. Pol., A 103, 325 (2003).

[23] P. Berberich and M. Schwarte, Z. Phys. B: Condens. Matter 64, 1 (1986). We use the static potential constant value of $2.1 \mathrm{eV}$ for Si:B given in Table 4, and the expression for the ground-state splitting energy $E$ as a function of stress $X$ along [001], given in Table 3. We also make use of the low-temperature (4-K) elastic constants for Si found in Table 1 of Ref. [32].

[24] P. Berberich and H. Kinder, J. Phys., Colloq. 42, C6-374 (1981).

[25] M. Schwarte and P. Berberich, J. Phys. C: Solid State Phys. 18, 3225 (1985).

[26] D. Fröhlich and W. Nieswand, Philos. Mag. B 70, 321 (1994).

[27] T. E. Wilson, Int. J. Infrared Millimeter Waves 14, 303 (1993).

[28] K. F. Renk and L. Genzel, Appl. Opt. 1, 642 (1962).

[29] C. T. Gross, J. Kiess, A. Mayer, and F. Keilmann, IEEE J. Quantum Electron. 23, 377 (1987).

[30] P. P. Woskov, V. S. Bajaj, M. K. Hornstein, R. J. Temkin, and R. G. Griffin, IEEE Trans. Microwave Theory Tech. 53, 1863 (2005).

[31] B. A. Auld, Acoustic Waves and Fields, 2nd ed. (Kreiger, Malabar, FL, 1990), Vol. 1.

[32] A. Berke, A. P. Mayer, and R. K. Wehner, J. Phys. C: Solid State Phys. 21, 2305 (1988).

[33] M. Born and E. Wolf, Principles of Optics, 6th ed. (Pergamon, Oxford, UK, 1980), p. 611.

[34] E. V. Loewenstein, D. R. Smith, and R. L. Morgan, Appl. Opt. 12, 398 (1973).

[35] S. B. Kaplan, J. Low Temp. Phys. 37, 343 (1979).

[36] J. P. Wolfe, Imaging Phonons: Acoustic Wave Propagation in Solids (Cambridge University Press, Cambridge, UK, 1998), p. 116. The slowness sheet for the LA mode possesses only convex curvature and cannot produce phonon-focusing caustics.

[37] C. Kittel, Introduction to Solid State Physics, 7th ed. (Wiley, New York, 1996), p. 108

[38] O. L. Muskens and J. I. Dijkhuis, Propagation and diffraction of picosecond acoustic packets in the soliton regime, in Optical Solitons, edited by K. Porsezian and V. C. Kariakose (Springer, Berlin, 2003), p. 391.

[39] A. Lamas-Linares, J. C. Howell, and D. Bouwmeester, Nature (London) 412, 887 (2001).

[40] D. Grassani, A. Simbula, S. Pirotta, M. Galli, M. Menotti, N. Harris, T. Baehr-Jones, M. Hochberg, C. Galland, M. Liscidini, and D. Bajoni, Sci. Rep. 6, 23564 (2016). 